\title{
PREDICTORS OF BRONCHOPULMONARY DYSPLASIA (BPD) IN A LOW BPD SETTING:A 6- YEAR POPULATION-BASED COHORT OF THE MARCHE REGION
}

\author{
M.L. Palazzi ${ }^{1}$, C. Proietti Pannunzi ${ }^{1}$, M. Pasqualini ${ }^{1}$, C. Rondina ${ }^{1}$, I. Burattini ${ }^{1}$, R. D'Ascenzo ${ }^{1}$, P. Cogo $^{2}$, \\ V.P. Carnielli ${ }^{1}$

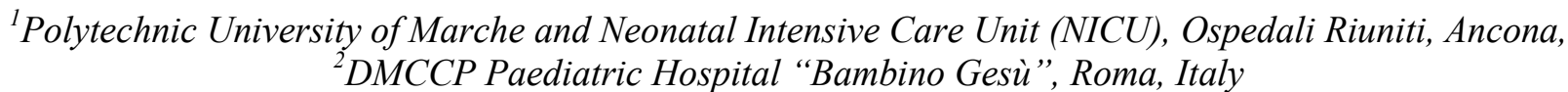

Background and objective: BPD remains a severe complication of premature birth. We studied the risk factors and early predictors of BPD.

Methods: All infants from 24,0 to 31,6 weeks born in the Marche Region from 2004 to 2009 were studied.

Results: 629 infants were eligible, with 69 deaths (11\%), 97 BPDs (15.4\%), 161 deaths and/or BPD (25.6\%). Histological chorioamnionitis (HCA) were $12,3 \%$ and culture proven sepsis $13.7 \%$. Gestational age(GA), birth weight (BW),BW standard deviation score (BW-SDS),duration of mechanical ventilation and continuous positive airway pressure, oxygen supplementation, intubation within 6 hours from birth, ventilation associated pneumonia, patent ductus arteriosus (PDA), sepsis, respiratory distress syndrome (RDS), surfactant treatment (number of doses, age at $1^{\text {st }}$ dose, oxygenation-index (OI) and FiO2 before the $1^{\text {st }}$ dose), pulmonary hypertension, inhaled nitric oxide (iNO), were all significant at the univariate analysis. We found no significant association between BPD and HCA or early-onset sepsis. Multivariate logistic regression analysis is reported in table below.

\begin{tabular}{|l|c|c|c|}
\hline Risk Factors & $\mathrm{p}$ & OR & CI \\
\hline GA & 0,000 & 0,954 & $0,937-0,971$ \\
\hline BW-SDS & 0,006 & 0,708 & $0,553-0,906$ \\
\hline PDA & 0,003 & 0,432 & $0,250-0,745$ \\
\hline age at $1^{\text {st }}$ surfactant & 0,004 & 0,956 & $0,916-0,997$ \\
\hline OI pre-1 ${ }^{\text {st }}$ surfactant & 0,000 & 1,103 & $1,045-1,166$ \\
\hline
\end{tabular}

[Table]

Conclusion: In our cohort of preterm infants receiving "optimal" prenatal care with a low rate of complications, BPD percentage was low and significantly associated with RDS severity and PDA but not with sepsis and/or HCA. 